\title{
FACTORES CRÍTICOS PARA EL DESARROLLO EXITOSO DE LOS CENTROS ODONTOLÓGICOS. CASO: TACNA (PERÚ)
}

\section{CRITICAL FACTORS FOR THE SUCCESSFUL DEVELOPMENT OF DENTAL CARE CENTERS. CASE: TACTA (PERÚ)}

Jorge Bernal $^{1}$

Ernesto Leo ${ }^{2}$

1. Doctor en Financiación e Investigación Comercial (UAM-España), académico de la Escuela Universitaria de Administración y Negocios de la Universidad de Tarapacá (Arica-Chile). E-mail: jbernal@uta.cl

2. Magister en Dirección y Gestión de Empresas (UTA-Chile), académico de la Escuela de Postgrado Neumann Business School (Tacna-Perú). E-mail: eleo@neumann.edu.pe

Citación sugerida:

Bernal, J. y Leo, E. (2017). Factores críticos para el desarrollo exitoso de los centros odontológicos. Caso: Tacna (Perú). 3C TIC: Cuadernos de desarrollo aplicados a las TIC, 6(4), 42-53. DOI: $<$ http://dx.doi.org/10.17993/3ctic.2017.58.42-53/>. 


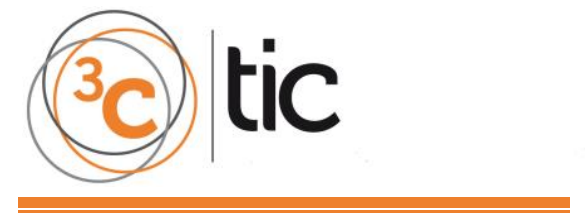

\section{RESUMEN}

La salud bucal forma parte de la salud general, siendo reconocida como un componente esencial para la calidad de vida de los ciudadanos. A consecuencia de ello, existe una proliferación de centros odontológicos, tal es el caso de la ciudad de Tacna (Perú). En esta ciudad, existe un mercado dinámico y competitivo, principalmente por la llegada de gran número de turistas chilenos que demandan atención bucal, representando cerca del $70 \%$ de sus clientes. Sin embargo, se desconoce qué factores inciden para que algunas organizaciones tengan éxito y otras no. La presente investigación tiene como objetivo determinar los factores críticos de éxito de los centros odontológicos y cuales pueden considerarse como una ventaja competitiva sostenible para ellos. Para alcanzar este objetivo, se realizó una rigurosa revisión bibliográfica, se encuestó a los dueños y/o administradores de los 74 centros odontológicos existentes en la ciudad de Tacna y se entrevistó a los actores más relevantes. El estudio concluyó que los centros odontológicos de la ciudad de Tacna (Perú) consideran como los factores de éxito más relevantes: la dirección de empresa, los recursos financieros, el control interno y los factores sociales.

\section{ABSTRACT}

Oral health is part of the general health, being recognized as an essential component for the quality of life of the citizens. As a result, there is a proliferation of dental care centers, such as the case in the city of Tacna (Peru). In this city, there is a dynamic and competitive market, mainly due to the arrival of large numbers of Chilean tourists who demand dental care attention, representing about $70 \%$ of their clients. However, it is not known what factors influence for some organizations to succeed and others to fail. The present research aims to determine the critical success factors of dental care centers and which can be considered as a sustainable competitive advantage for them. To reach this objective, a rigorous bibliographical review was carried out, the owners and / or administrators of the $\mathbf{7 4}$ dental centers in the city of Tacna were interviewed and the most relevant actors were interviewed. The study concluded that the dental centers of the city of Tacna (Peru) consider as the most relevant factors of success: business management, financial resources, internal control and social factors.

\section{PALABRAS CLAVE}

Factores críticos, odontología, ventaja competitiva, salud bucal, estrategia.

\section{KEY WORDS}

Critical factors, odontology, competitive advantage, dental health, strategy. 


\section{INTRODUCCIÓN}

Con la globalización los centros odontológicos han tenido que romper esquemas y salirse de los sistemas y métodos tradicionales de funcionamiento, las exigencias de los consumidores han cambiado a través de los años, por tal motivo las organizaciones se han visto en la necesidad de modernizar sus procesos e instalaciones para poder atraer a un mayor número de clientes.

Las empresas de hoy deben mantener una estrecha relación con el cliente externo e interno, dicho esto en otras palabras, los empleados de una organización deben de tener clara la misión, visión, valores y principios fundamentales de la organización para que la filosofía empresarial sea transmitida de una manera correcta a los usuarios. Es un desafío que el capital humano de una organización sea el adecuado para el óptimo funcionamiento de la misma. Por otra parte es de suma importancia conocer al consumidor pues hoy en día demanda una atención personalizada, por tanto se debe tener plenamente identificado las pretensiones de los clientes para que la estrategia de comunicación y atención sea la adecuada con cada tipo de cliente y así conseguir un alto posicionamiento y un buen nivel de retroalimentación (Calero et al., 2008).

Por tanto, las organizaciones deben estar orientadas al consumidor pero no de una manera masiva, por el contrario, se debe presentar unas opciones personalizadas enfocadas en las diferentes necesidades que presentan los diferentes segmentos de clientes, en el mismo sentido se debe tener claro lo que la organización quiere brindar y transmitir para de esta manera prestar un servicio integral de calidad (Haynes, 2013).

Es así, como las empresas deben establecer estrategias que las hagan perdurables en el tiempo y adaptables ante los constantes cambios que se presentan con la globalización. Poseer ventajas competitivas, procesos adecuados con estándares de calidad y seguimiento, de manera de garantizar un mejor desarrollo organizacional y por tanto la prestación de un mejor servicio, por ende una mayor conexión con los diferentes tipos de clientes (Trkman, 2010).

Para una organización es de vital importancia tener clara las estrategias que quiere implementar para alcanzar sus objetivos pero, es más importante establecer el cómo se van a implementar las estrategias para que sean altamente efectivas. En este caso conocer las prioridades de una empresa es un factor clave para determinar el contexto en el que se va a trabajar para lograr de una mejor manera los propósitos de una organización, para ello es importante establecer métodos de control rigurosos que permitan conocer los avances e impacto de cada proceso.

Poseer una visión hacia el futuro, va a permitir ser más adaptables a los cambios y alcanzar ventajas competitivas sostenibles de una manera rápida según se presenten las necesidades y también reconocer con claridad cuáles son los factores internos y externos que afectan el desarrollo exitoso de la organización. Estos factores son conocidos como los factores críticos de éxito (FCE), que es el término utilizado para describir una actividad que permita asegurar el éxito de una organización (Pinto, 2000). 
Si bien, los FCE han sido investigados para diferentes tipos de organización, también es cierto, que en el caso de los centros odontológicos no han sido estudiados. Por lo anterior, la presente investigación tiene como objetivo determinar los factores críticos de éxito de los centros odontológicos y cuales pueden considerarse como una ventaja competitiva sostenible para ellos. Para ello, el estudio encuestó a los dueños y/o administradores de los 74 centros odontológicos existentes en la ciudad de Tacna (Perú) y se entrevistó a los actores más relevantes.

\section{METODOLOGÍA}

Los indicadores fueron clasificados en dos tipos: externos e internos. Los primeros son exógenos, es decir la organización es influenciada por ellos pero no determina su comportamiento; y los segundos son endógenos y por tanto son controlados directamente por la organización.

Para determinar cuáles son los factores que inciden en el éxito de la empresa se determinó usar un cuestionario de 52 preguntas que se relacionaban con 7 factores externos y 8 factores internos, utilizando la escala de Likert en un intervalo de 1 (No importa) al 5 (Muy importante).

Villajuana (2010) menciona que el análisis externo responde a cuatro preguntas:

- ¿Qué podría hacer?: Sugiere que deben conocerse los frenos e impulsos que tienen efectos sobre los propósitos particulares de la unidad estratégica o tienen trascendencia interna. Comprende los acontecimientos político-legales, económicos, tecnológicos y competitivos del sector donde actúa un negocio.

- ¿Qué debería hacer?: Se refiere a la necesidad de considerar las expectativas de la sociedad en el ámbito del respeto de valores morales, derechos sociales y preservación del medio ambiente. Su consideración por la unidad estratégica conlleva la búsqueda de la verdadera trascendencia, es decir aquella que en términos de tiempo y espacio traspasa los intereses organizacionales internos.

- ¿Qué deseo hacer?: Lleva al estudio de las capacidades emocionales y espirituales de las personas que van más allá de su potencial racional. En concreto, alcanza la deducción de fortalezas y debilidades en los campos relacionados a la motivación, confianza, compromiso y al cultivo de valores.

- ¿Qué puedo hacer?: Esta pregunta conduce a la exploración de las fortalezas y debilidades asociadas a las capacidades racionales de los recursos de la unidad estratégica. Estas capacidades por lo general son mensurables y tangibles.

Por su parte Porter (1982) a través de su conocida estructura, llamada el modelo de las cinco fuerzas, ayuda a los administradores a realizar este análisis. Su modelo se enfoca en las cinco 
fuerzas que conforman la competencia en una industria: 1) el riesgo de que entren nuevos competidores, 2) la intensidad de la rivalidad entre las compañías establecidas en una industria, 3) el poder de negociación de los compradores, 4) el poder de negociación de los proveedores y 5) la

cercanía de los sustitutos para los productos que ofrece una industria.

\begin{tabular}{|c|c|c|c|}
\hline $\begin{array}{c}\text { Matriz de } \\
\text { operacionalización } \\
\text { de la } \\
\text { variableVARIABLE }\end{array}$ & DIMENSIONES & INDICADORES & $\begin{array}{l}\text { Nivel de } \\
\text { medición }\end{array}$ \\
\hline \multirow{16}{*}{$\begin{array}{l}\text { FACTORES } \\
\text { CRÍTICOS }\end{array}$} & \multirow{8}{*}{$\begin{array}{l}\text { FACTORES CRÍTICOS } \\
\text { EXTERNOS }\end{array}$} & Factores políticos y legales & \multirow{8}{*}{ Ordinal } \\
\hline & & Economía Nacional & \\
\hline & & Factores Sociales & \\
\hline & & & \\
\hline & & Tecnología disponible & \\
\hline & & Competencia del sector & \\
\hline & & Calidad de los proveedores & \\
\hline & & Calidad de los clientes & \\
\hline & \multirow{8}{*}{$\begin{array}{l}\text { FACTORES CRÍTICOS } \\
\text { INTERNOS }\end{array}$} & Planificación a futuro & \multirow{8}{*}{ Ordinal } \\
\hline & & Organización interna & \\
\hline & & Integración de conocimientos & \\
\hline & & Dirección de la empresa & \\
\hline & & Control interno & \\
\hline & & Procesos eficientes & \\
\hline & & Atención a clientes & \\
\hline & & Recursos Financieros & \\
\hline
\end{tabular}

Tabla 1. Matriz de factores críticos.

Fuente: elaboración propia.

Los factores externos considerados fueron: Factores Políticos y Legales (2 Preguntas); Factores Económicos (2 Preguntas); Factores Sociales (2 Preguntas); Factores Tecnológicos (3 
Preguntas); Competencia del Sector (2 Preguntas); Relación con los Proveedores (2 Preguntas); Relación con los Clientes (3 Preguntas).

Según Villajuana (2010), los indicadores internos son aquellos que sirven para medir la capacidad de los procesos clave y de las dimensiones que representan los objetivos del diagnóstico interno. Por lo general se expresan en términos de eficacia (relación entre la meta real alcanzada y la meta planeada), eficiencia (esfuerzo medido en términos de tiempo, costo y/o productividad) y efectividad (eficiencia más eficacia).

Los factores Internos considerados fueron: Planificación a Futuro (4 Preguntas); Organización Interna (3 Preguntas); Integración y Aprendizaje de Conocimientos (7 Preguntas); Dirección y Liderazgo (4 Preguntas); Control Interno (2 Preguntas); Procesos Internos Eficientes (8 Preguntas); Capacidad de Respuesta y Calidad como confianza y excelencia (5 Preguntas) y Recursos Financieros (3 Preguntas).

Para poder obtener la información relevante, se determinó aplicar las encuestas a los Dueños y/o Administradores de los centros odontológicos, ya que ellos son quienes que se encargan de la operatividad de las organizaciones (Hurtado et al., 2016).

Durante el mes de octubre y noviembre del 2016 se visitaron los 74 centros odontológicos ubicados en la ciudad de Tacna, provincia de Tacna, distrito de Tacna, para aplicar la encuesta, resaltando la calidad de confidencial de la información para obtener el mayor nivel de veracidad de las respuestas por parte de los dueños y/o Administradores de los Centros.

\section{DESARROLLO}

En Perú, uno de los sectores considerados de gran relevancia para la calidad de vida de sus ciudadanos es la salud y específicamente la salud dental. Tacna es una ciudad ubicada al sur de Perú, en su frontera con el vecino país de Chile y a $60 \mathrm{Km}$. de la ciudad de Arica (ciudad más al norte de Chile), aproximadamente llegan entre 10,000 a 15,000 chilenos diariamente a Tacna gastando un promedio de 60 dólares por persona al día, es por ello que existe una fuerte demanda de servicios diversos en la ciudad a costos competitivos y que les parecen atractivos dentro de los cuales se encuentran los servicios gastronómicos y los servicios médicos.

El crecimiento de los servicios médicos durante los últimos años, se debe a que los pacientes de nacionalidad chilena consideran que la atención médica en Tacna es superior a la de su país, y que el costo por atención es menor. Dentro de la gama de la oferta de salud que se ofrece en la ciudad, uno de los servicios que más ha crecido es el de servicios odontológicos, logrando que las tradicionales oficinas de dentistas independientes se asocien y se convirtieran en centros odontológicos especializados, los cuales han venido creciendo no solo en cantidad sino en capacidad instalada.

Asimismo el sector odontológico ha visto un cambio mayor en estos últimos años con la llegada de nueva y sofisticada tecnología que ha obligado a los dentistas a esmerarse por la 


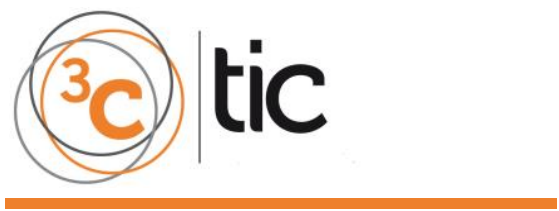

demanda de los pacientes que quieren verse mejor y lucir sonrisas con cambios radicales y en menor tiempo, guiados muchas veces por estereotipos vistos en televisión e internet.

Se determinó que los centros odontológicos que vienen operando en la ciudad de Tacna, cuentan con un promedio de 5 odontólogos dentro de staff, distribuidos de la siguiente manera: un $24,4 \%$ de estos centros cuentan entre 1 a 2 odontólogos, el $25,7 \%$ cuentan entre 3 a 4 odontólogos, el 37,7\% cuentan entre 5 a 6 odontólogos y el 12,2\% cuentan entre 7 a más odontólogos (ver gráfico 1).

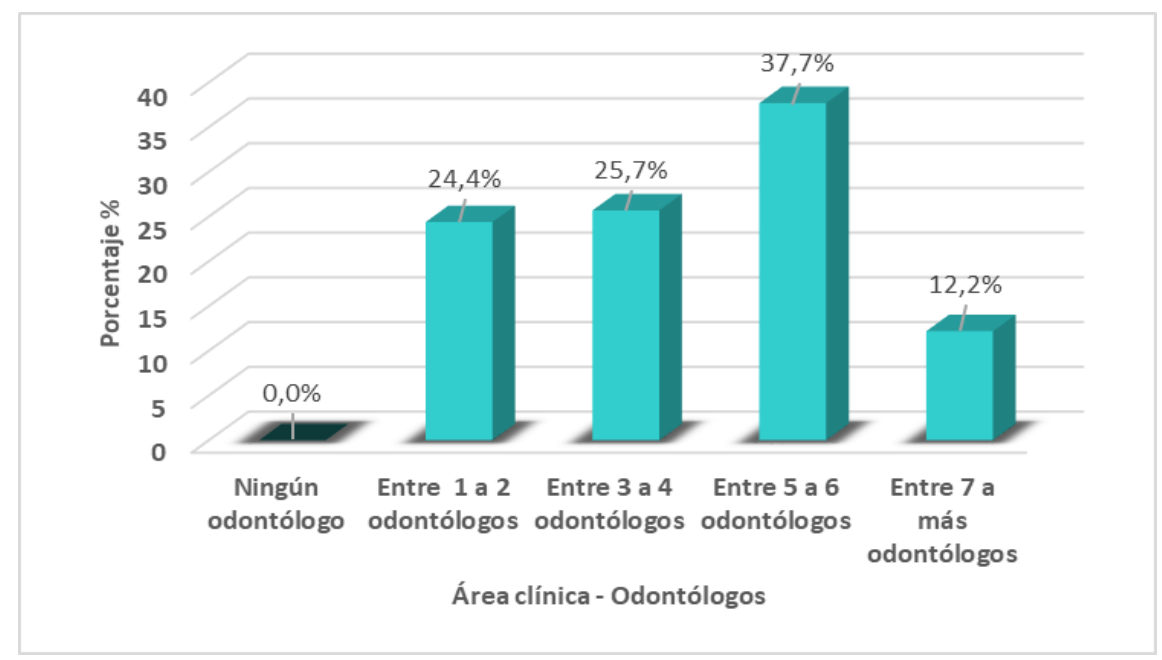

Gráfico 1. Número de odontólogos por clínica.

Fuente: elaboración propia.

Este equipo es numeroso debido a la alta demanda y al tiempo necesario que conlleva las atenciones dentales, lo que origina un promedio de atenciones entre 0 a 10 casos por día el $75,7 \%$ de las veces, un $13,5 \%$ atiende entre 11 y 20 casos por día y un $10.8 \%$ atiende más de 21 casos por día (ver gráfico 2 ).

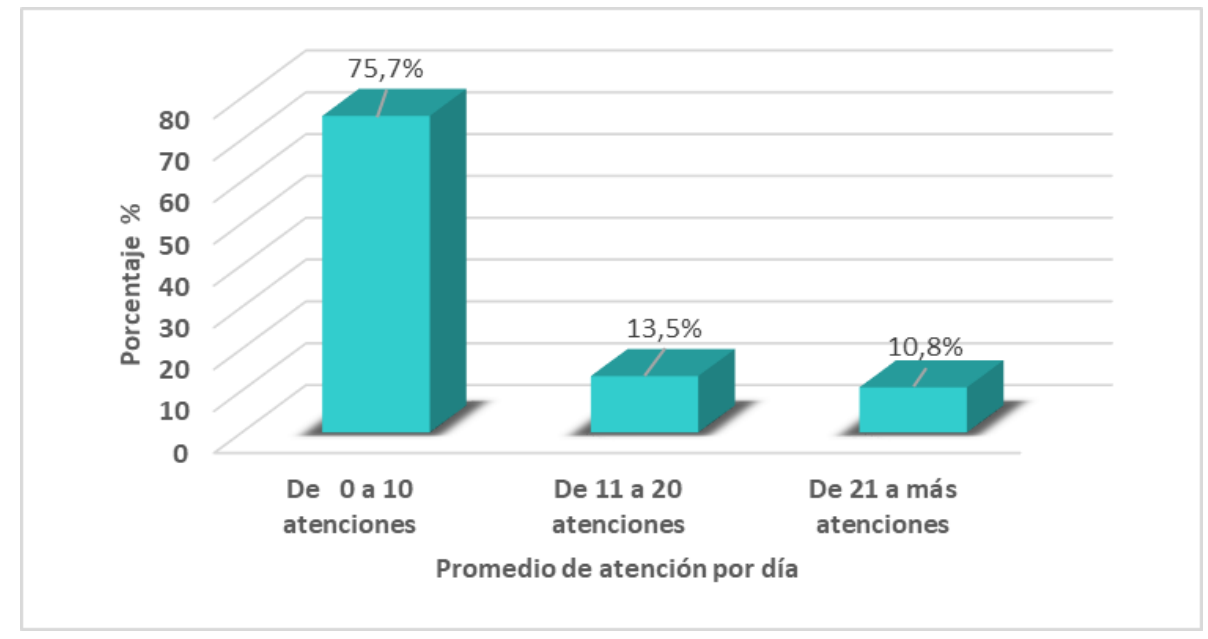

Gráfico 2. Promedio de atenciones por día.

Fuente: elaboración propia. 


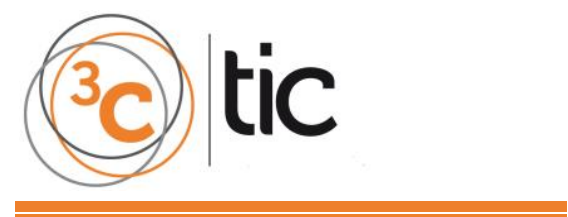

En Tacna, los ciudadanos del vecino país de Chile pueden encontrar servicios odontológicos hasta por la quinta parte de lo que cuesta en su país y si se hace una suma de todos los tratamientos que se requieren es una oferta altamente atractiva intervenirse en Tacna, ya que es posible encontrar consultorios y centros odontológicos de calidad.

El origen de los clientes de los centros odontológicos de la ciudad de Tacna en un 24,1\% proviene de la ciudad de Tacna, mientas que un $72,8 \%$ proviene del vecino país de Chile, de otras ciudades del Perú en un $2,6 \%$ y un $0,5 \%$ de otros países (ver gráfico 3 ).

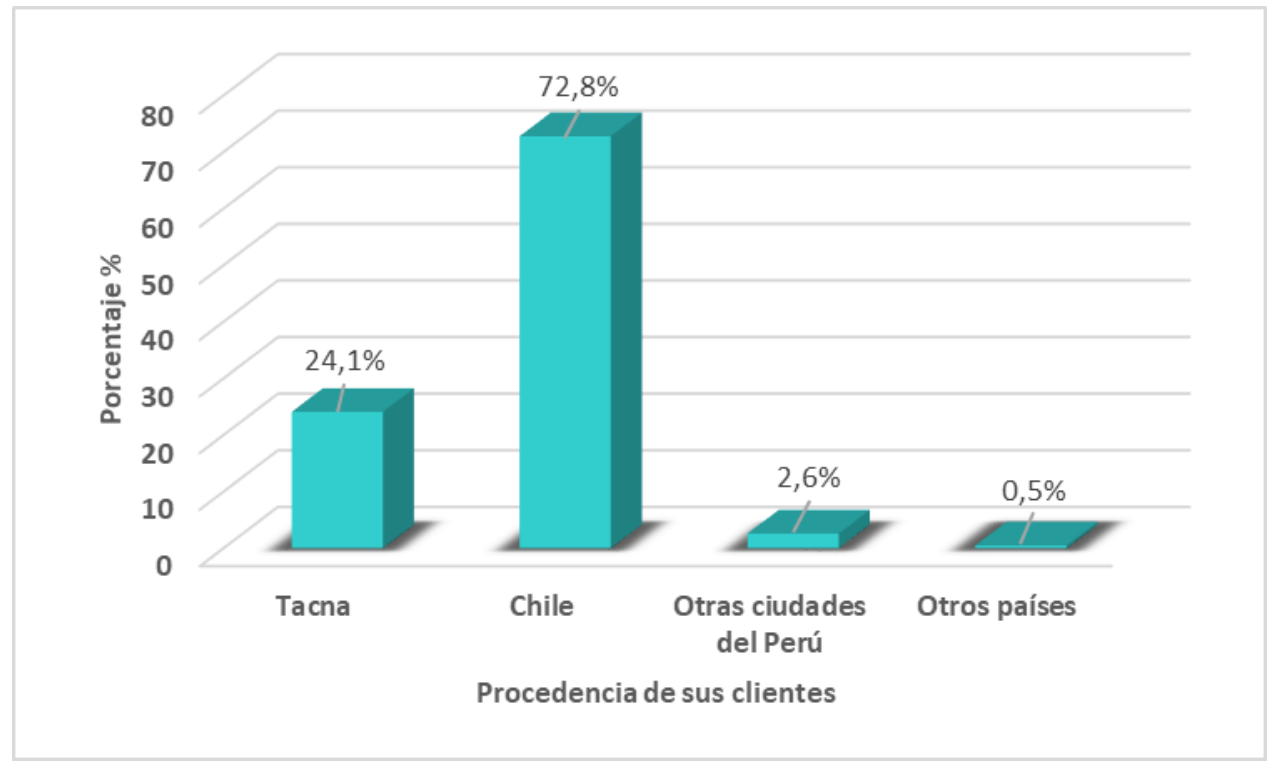

Gráfico 3. Procedencia de los clientes.

Fuente: elaboración propia.

Debido a esto los centro odontológicos en Tacna están trabajando por mejorar cada vez para satisfacer a los clientes, logrando algunos de estos centros odontológicos destacarse sobre la oferta regular del medio, la pregunta a resolver consiste en determinar cuáles son los factores que inciden en que algunas organizaciones se destaquen frente a otras.

Por lo antes referido, la investigación pretende contribuir al conocimiento, determinando cuáles son los factores de éxito que las empresas odontológicas consideran son las responsables del éxito en la ciudad de Tacna, cuyas características son tan particulares, a fin de renovar y dar un aporte al conocimiento existente y poder mejorar la comprensión y desarrollo de los factores de éxito que permitirán obtener ventajas competitivas al sector de servicios odontológicos, permitiéndoles invertir en estrategias que agreguen valor a los clientes que hacen uso de este servicio.

Según Villajuana (2010), el propósito del análisis estratégico es decidir qué hacer frente a los indicadores determinantes del éxito y éste es directamente proporcional a la precisión con la que se exploren los indicadores externos e internos más importantes. A su vez dicha exactitud dependerá de la eficacia en la ponderación de la incidencia de cada indicador externo o interno (Alonso, 2010). 


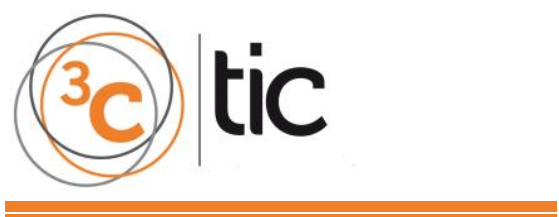

En el presente trabajo de investigación se toma la definición dada por Rockart (1979) quien define FCE como:

"Los FCE son, para cualquier negocio, un número limitado de áreas en las que los resultados, si éstos son satisfactorios, garantizarán un rendimiento competitivo exitoso para la organización. Estas son áreas principales donde las cosas deben ir bien para que el negocio florezca: si los resultados en dichas áreas no son los adecuados, los esfuerzos de la organización, para ese período, no estarán definidos y los objetivos de gestión no podrán ser alcanzados (p. 85)".

\section{RESULTADOS}

El resultado de los factores externos son: Factores sociales $(4,72)$, Factores Tecnológicos $(4,68)$, Relación con los proveedores $(4,34)$, factores políticos y legales $(4,01)$, Factores Económicos $(3,68)$ y competencias del sector $(3,05)$, (ver gráfico 4$)$.

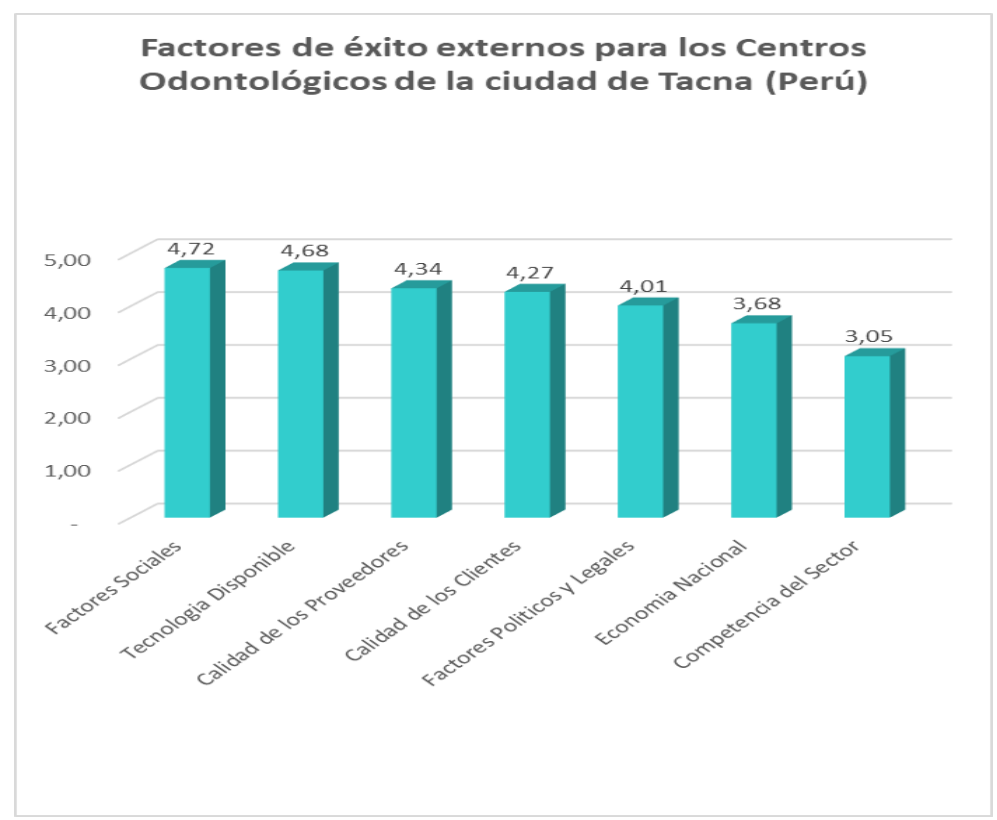

Gráfico 4. Factores críticos externos de éxito.

Fuente: elaboración propia.

El orden de los factores internos son: dirección de las empresas $(4,81)$, recursos financieros $(4,77)$, control internos $(4,73)$, atención al cliente $(4,69)$, organización interna $(4,55)$, planificación a futuro $(4,53)$, integración de conocimientos $(4,42)$ y procesos eficientes $(4,42)$, (ver gráfico 5). 


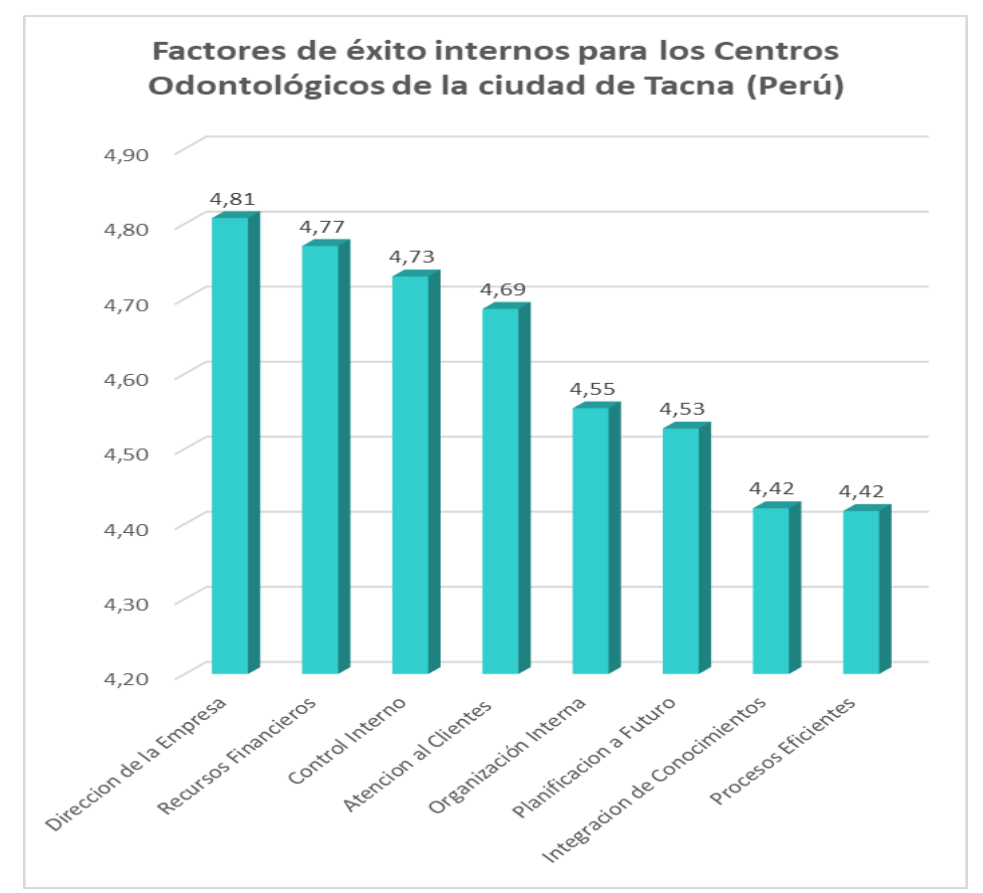

Gráfico 5. Factores críticos internos de éxito. Fuente: elaboración propia.

Los factores considerados como los más determinantes en el éxito por los dueños y/o administradores de las empresas odontológicas de la ciudad de Tacna fueron los siguientes: Dirección de la Empresa (4.81); Recursos Financieros (4.77); Control Interno (4.73); Factores Sociales (4.72); Capacidad de Respuesta al Cliente (4.69); Factores Tecnológicos (4.68); Organización Interna (4.55); Planificación a Futuro (4.53); Integración y Aprendizaje de conocimientos (4.42); Procesos Internos Eficientes (4.42); Relación con los Proveedores (4.34); Relación con los Clientes (4.01); Factores Políticos y Legales (4.01); Factores Económicos (3.68) y Competencia del Sector (3.05), (ver gráfico 6). 


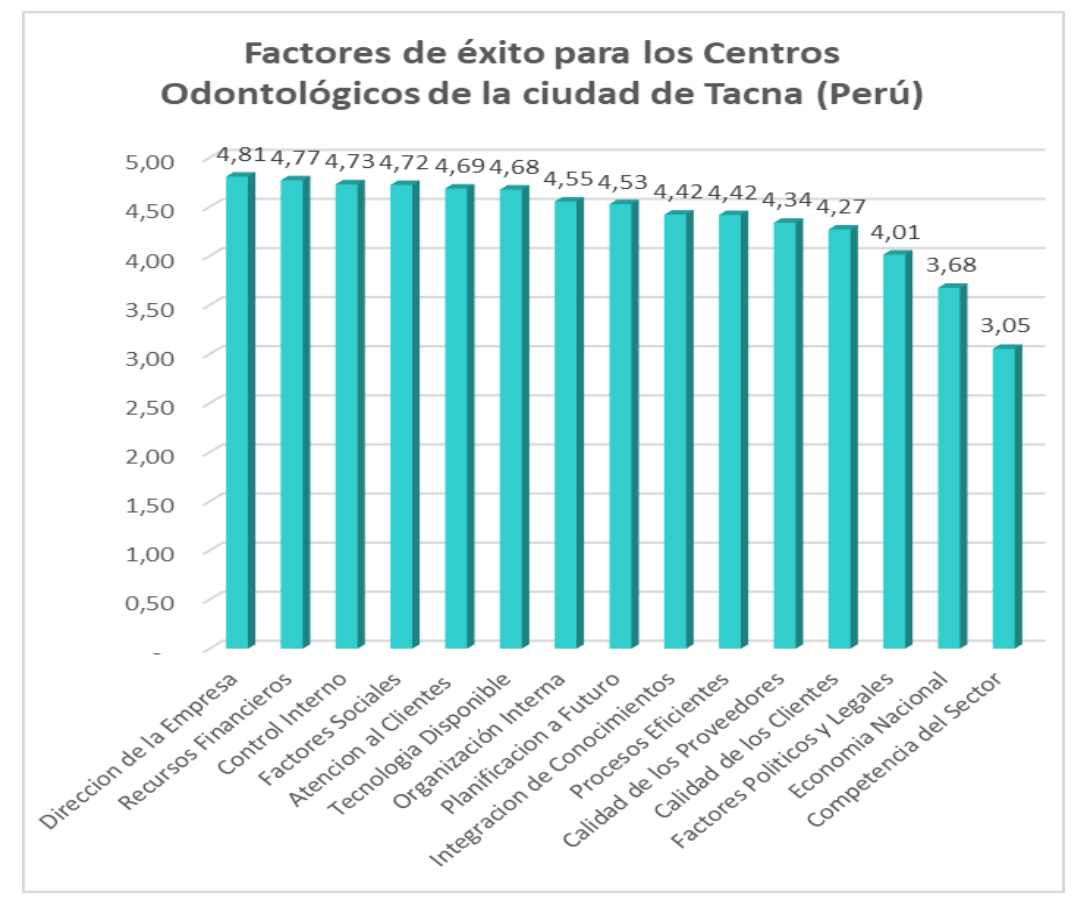

Gráfico 6. Factores críticos éxito. Fuente: elaboración propia.

\section{CONCLUSIONES}

La detección de los factores críticos de éxito en una organización, permite la priorización de las actividades que se deben alcanzar para el éxito del negocio. Es así, como esta investigación permitió determinar qué factores deben ser especialmente observados para el caso de los centros odontológicos. Queda demostrado que los factores de éxito internos son más importantes que los externos para los centros odontológicos, habiendo obtenido los factores internos un promedio superior (4.61) en comparación a los externos (4.11).

Además, el estudio concluyó que los centros odontológicos de la ciudad de Tacna (Perú), consideran como los factores de éxito más relevantes: la dirección de empresa, los recursos financieros, el control interno y los factores sociales. 


\section{REFERENCIAS BIBLIOGRÁFICAS}

Alonso, V. (2010). Factores críticos de éxito y evaluación de la competitividad de destinos turísticos. Estudios y perspectivas en turismo, 19(2), 201-220.

Calero, J. \& Rodríguez, J. (2008). La calidad y el mejoramiento continuo: claves en el éxito del ejercicio profesional en odontología. Colombia Medica, 39(1), 11-27.

Haynes, S. (2013). A perspective from the dental industry on minimum intervention dentistry. Australian Dental Journal, 58(1), 66-69.

Hill, C. \& Jones, G. (2011). Administración Estratégica: Un enfoque integral. México: Cengage Learning Editores.

Hurtado, A., Negreiros, S. y Villanueva, A. (2016). Determinación de los factores críticos para el desarrollo de las empresas odontológicas de la ciudad de Tacna. Caso: Tejada centro odontológico especializado EIRL. Tesis de master. Escuela de Postgrado Neumann Business School.

Pinto, J. (2000). Asignación y determinación de prioridades de procesos esenciales, con base en los factores críticos de éxito. Estudios Gerenciales, 74(1), 79-89.

Porter, M. (1982). Estrategia competitiva: técnicas para el análisis de los sectores industriales y de la competencia. México: Editorial S.A. de C.V.

Rockart, J.F. (1979). Chief executives define their own data needs. Harvard Business Review, 57(1), 81-92.

Trkman, P. (2010). The critical success factors of business process management. International Journal of Information Management, 30(1), 125 - 134.

Villajuana, C. (2010). Gestión Estratégica Integral. Lima: Villajuana Consultores S.A.C. 